\title{
Three Perspectives: The Approach of Neoadjuvant Treatment of Rectum Cancer According to Medical Oncologists, Radiation Oncologists, and Surgeons
}

Ismail Beypinar ( $\square$ ibeypinar@yahoo.com )

Eskisehir City Hospital

Mustafa Tercan

Eskisehir City Hospital

Fuzuli Tugrul

Eskisehir City Hospital

\section{Research Article}

Keywords: Chemotherapy, Neoadjuvant Treatment, Radiotherapy, Rectal Cancer, Treatment Modalities

Posted Date: March 29th, 2022

DOI: https://doi.org/10.21203/rs.3.rs-1236318/v2

License: () (1) This work is licensed under a Creative Commons Attribution 4.0 International License. Read Full License 


\section{Abstract}

\section{Purpose}

Two treatment options are considered for radiotherapy: short-course radiotherapy and immediate surgery or chemoradiation with 5-Fluorouracil based chemotherapy with delayed surgery. We try to evaluate the real-life treatment approaches of medical, radiation, and surgical oncologists for neoadjuvant treatment of rectal cancers.

\section{Method}

The online survey form was established via Google Forms. The survey was taken voluntarily by medical oncologists, radiation oncologists, surgical oncologists, and general surgeons.

Results

One hundred eighty-three of the participants were medical oncologists while 36 were radiotherapists and 36 were surgeons. Most of the study population preferred long-course radiation therapy and chemotherapy (85\%). Two-thirds of the participants preferred chemotherapy before operation. The most frequent chemotherapy cycles for the pre-operative setting were 'three' or 'four-or-more'. $(27,8 \%$ and $25,1 \%$ respectively) Medical oncologists had a significantly higher tendency of offering chemotherapy between radiation therapy and surgery compared with the other groups. The optimal time of surgery was different between groups. There was no difference among groups between surgery and the 'watch \& wait' strategy. Neoadjuvant chemotherapy regimens were different between groups significantly.

\section{Conclusion}

We found the new pre-operative chemotherapy regimen with short-course radiotherapy was slowly adopted in current practice. Also, medical oncologists tend pre-operative chemotherapy compared with other groups.

\section{Introduction}

Chemoradiation (CRT) is the gold standard of care in newly diagnosed rectal cancers.[1] The main objective of the treatment is to improve surgical outcomes, prevent local recurrence, prolong disease-free (DFS) and overall survival (OS). Two treatment options are considered for radiation therapy: short-course radiotherapy (RT) and immediate surgery or chemoradiation with 5-Fluorouracil (5-FU) based chemotherapy (CT) with delayed surgery.[1, 2] Especially in controversial areas, the initial treatment plan is mainly depending on the decision of the treating physician.[3] Two treatment options have similar results in terms of survival, R0 surgery, distant and local recurrences. Pathological complete remission (pCR) rates are higher in long course radiotherapy in combination with CT.[4] The local and distant recurrence rates were favoring $\mathrm{CR}$ after $\mathrm{CRT}$ compared with non-responders after surgery.[5] Prior studies showed that the addition of multidrug (only oxaliplatin-containing) regimens was not related to increased 
pCR but resulted in higher rates of toxicity. A modest benefit was observed with the addition of oxaliplatin to 5 -FU and radiation.[6]

Rectal cancers with mid or low location (infraperitoneal) which are T3-4 or with nodal metastasis, are recommended to receive CRT by current guidelines. Also, circumferential resection margin (CRM) involvement is an indication for neoadjuvant treatment. Possible total neoadjuvant treatment may be offered to current guidelines including the FOLFOXIRI regimen.[7] Although CRT was associated with decreased local recurrence, no improvement was observed in OS.[1, 8] CRT mainly resulted in downsizing in the majority of the patients (70\%), even less with a pCR (20\%). The pCR group is related to a good prognosis and has an excellent OS of over 90\%.[9] Although small tumors have a better response to CRT, there are multiple controversial factors determining CRT response. The optimal interval after CRT to surgery is still unknown. The Lyon trial compared 2 weeks delay with 6 weeks after CRT which resulted in increased $\mathrm{PCR}$ and near $\mathrm{PCR}$ rates.[10] The main objective of the delay after CRT depends on the delayed lysis of the tumor cells after immediate DNA damage with CRT. The tumor cells were reported to be morphologically intact shortly after RT.[11, 12] Multiple studies evaluated the optimal time for the surgery after CRT, but there was no correlation between studies.[13-15]

In a Canadian study evaluating the surgical attitudes on rectal cancer, the waiting period after CRT was mostly determined to be 6 weeks. Also, low numbers of the 'Watch \& Wait' strategy were observed. This study was mainly focused on surgical techniques, not the selection of the type of treatment. Different surgical types were investigated in this study such as microscopic anal resection or total mesorectal surgery.[16] Also, a survey study was performed in the Netherlands to acquire information about the preferences and awareness of surgeons, especially in lateral lymph nodes status.[17]

In radiological studies, the tumor position, maximum distance from the anal verge, maximum tumor length, thickness, area, and volume were evaluated. These factors were reported to be a directive marker for pCR except for tumor thickness.[18] Different treatment preferences especially in controversial areas in colon cancer were previously studied. $[19,20]$

In this study, we try to evaluate the real-life treatment approaches of medical, radiation, and surgical oncologists for neoadjuvant treatment of rectal cancers.

\section{Materials And Methods}

\section{Participants}

An online survey link was sent to the medical oncologists, radiation oncologists, and surgeons via e-mail and mobile applications. The survey was started on $1^{\text {st }}$ November 2021 and ended on $29^{\text {th }}$ November 2021. The survey was sent to all oncologists, radiotherapists, and surgeons registered to their professional association in Turkey. 


\section{Survey}

The online survey form was established via Google Forms. The survey was answered voluntarily by medical oncologists, radiation oncologists, surgical oncologists, and general surgeons. At the beginning of the questionnaire, the participants were informed that their preferences in rectal cancer treatment would be evaluated. Their consent was acquired at the start of the survey. The participants were informed to answer the questions in optimal conditions such as treatment options and imagining methods. The survey was containing 14 questions that were designed to understand the participants' experience, working conditions, and rectal cancer treatment decisions. Two questions were mandatory for medical oncologists, not the other participants. Information about the experience in oncology practice, academic status, and the type of hospital was obtained from the participants. All questions were mandatory to answer.

\section{Statistical Analyze}

The survey results were analyzed using descriptive statistics, and Chi-square tests were used to calculate the $p$ values with SPSS version 21.0. Also, e-PICOS was used to analyze the difference between percentages by Z-test. The level of significance was determined as $p<0.05$.

\section{Ethics}

The study was carried out by the Declaration of Helsinki principles and all applicable regulations. The participants declared they fill the form voluntarily. There were no promotions or gifts to increase participation.

\section{Results}

The numbers of recipients for each group including medical oncologists, radiotherapists, and surgeons were 867,248 , and 217 respectively. A total number of 255 participants responded to the survey. One hundred eighty-three of the participants were medical oncologists while 36 were radiotherapists and 36 were surgeons. Nineteen of the surgeons were surgical oncologists and while 17 were general surgeons. The ages of the participants were mostly between 30 to 40 years. $(61,2 \%)$ Nearly fifty percent of the participants had less than 5 five-year of experience in oncology practice. Most of the study population was fellows and specialists with a cumulative percentage of 75 . Forty-six percent of the answerers were working in university hospitals. Nearly fifty percent of the participants had examined 5 or fewer newly diagnosed rectal cancers. Most of the study population preferred long-course radiation therapy and chemotherapy. (85\%) Two-thirds of the participants prefer chemotherapies before operation. The most frequent chemotherapy cycles for the pre-operative setting were 'three' or 'four-or-more'. ( $27,8 \%$ and $25,1 \%$ respectively) Forty percent of the participants preferred the XELOX protocol. Fifty-four of the medical oncologists prefer adjuvant chemotherapy even if the patient had complete remission after neoadjuvant 
treatment. Nearly half of the study participants considered the $7^{\text {th }}$ and $8^{\text {th }}$ weeks to be the optimal time for surgery. Most of the study population prefer surgery even if the patient had complete remission after neoadjuvant treatment. (82\%) The features of the study population were described in Table-1.

Table-1: Features of the study population

\begin{tabular}{|c|c|c|c|c|c|}
\hline Profession & $\begin{array}{l}\text { Medical } \\
\text { oncologists }\end{array}$ & $\begin{array}{l}\text { Radiation } \\
\text { oncologists }\end{array}$ & Surgeon & & \\
\hline$N(\%)$ & $184(72,2)$ & $36(14,1)$ & $35(13,7)$ & & \\
\hline Age (years) & $30-40$ & $41-50$ & $51-60$ & $61-70$ & \\
\hline$N(\%)$ & $156(61,2)$ & $77(30,2)$ & $18(7,1)$ & $4(1,6)$ & \\
\hline Experience (years) & 5 or less & $6-10$ & $11-20$ & $21-30$ & \\
\hline$N(\%)$ & $122(47,8)$ & $49(19,2)$ & $67(26,3)$ & $17(6,7)$ & \\
\hline Position & Fellow & Specialist & Assoc. Prof & Professor & \\
\hline$N(\%)$ & $97(38)$ & $95(37,3)$ & $31(12,2)$ & $32(12,5)$ & \\
\hline Facility & State $\mathrm{H}$. & Res. \& Edu H. & $\begin{array}{l}\text { University } \\
\text { H. }\end{array}$ & $\begin{array}{l}\text { Private } \\
\text { H. }\end{array}$ & \\
\hline$N(\%)$ & $21(8,2)$ & $81(31,8)$ & $118(46,3)$ & $32(12,7)$ & \\
\hline $\begin{array}{r}\text { (Monthly) Rectal } \\
\text { Cancer }\end{array}$ & 5 or less & $6-10$ & 11 or more & & \\
\hline$N(\%)$ & $123(48,2)$ & $91(35,7)$ & $41(16,1)$ & & \\
\hline Neo-adj treatment & Short-course & Long-course +CT & & & \\
\hline$N(\%)$ & $37(14,5)$ & $218(85,5)$ & & & \\
\hline $\begin{array}{r}\text { Neo-adj } \\
C T\end{array}$ & Yes & No & & & \\
\hline$N(\%)$ & $157(61,6)$ & $98(38,4)$ & & & \\
\hline Neo-Adj Cycles & 0 & 1 & 2 & 3 & 4 or more \\
\hline$N(\%)$ & $40(15,7)$ & $50(19,6)$ & $30(11,8)$ & $71(27,8)$ & $64(25,1)$ \\
\hline Туре of CT & None & Capecitabine/5-FU & XELOX & Folfox & \\
\hline$N(\%)$ & $62(24,3)$ & $35(13,7)$ & $102(40)$ & $56(22)$ & \\
\hline Optimal time for Surg. & 6 w. or before & $7-8 \mathrm{w}$ & $9-10 \mathrm{w}$ & $11-12 \mathrm{w}$. & $\begin{array}{l}13 \mathrm{w} \text { or } \\
\text { later }\end{array}$ \\
\hline$N(\%)$ & $43(16,9)$ & $122(47,8)$ & $38(14,9)$ & $48(18,8)$ & $4(1,6)$ \\
\hline CR strategy & Surgery & Watch \& wait & & & \\
\hline$N(\%)$ & $210(82,4)$ & $45(17,6)$ & & & \\
\hline
\end{tabular}

H: Hospital; Res. \& Edu. H.: Research and Educational Hospital; CT: Chemotherapy; Neo-Adj: Neo-Adjuvant; 5FU: 5-Fluorouracil; XELOX: Oxaliplatin plus Capecitabine; Folfox: Oxaliplatin, Leucovorin, 5-Fluorouracil; W.:

Week; CR: Complete Remission

There was a difference between age groups in terms of specialization which showed medical oncologists had a younger age comparing other branches. $(p<0,001)$ Also, medical oncologists significantly had less experience when compared with radiation oncologists and surgeons. $(p<0,001)$ There were no fellows in radiation oncology and surgeon groups, which was nearly fifty percent in the medical oncology subset. 
$(p<0,001)$ There was no difference between groups using short or long-course radiation plus chemotherapy. $(p=0,09)$ Medical oncologists had a significantly higher tendency of offering chemotherapy between radiation therapy and surgery compared with the other groups. $(p<0,001)$ The optimal time of surgery was different between groups. $(p=0,006)$ (Table- 2$)$

Table-2: The optimal operation time according to groups.

\begin{tabular}{r|lll} 
Time of Surgery & Medical Oncologists & Radiation Oncologists & Surgeon \\
\hline 6 w of before & $36(\mathrm{a})$ & $2(\mathrm{a})$ & $5(\mathrm{a})$ \\
$7-8$ weeks & $93(\mathrm{a})$ & $16(\mathrm{a})$ & $13(\mathrm{a})$ \\
\cline { 2 - 4 } $9-10$ weeks & $21(\mathrm{a})$ & $12(\mathrm{~b})$ & $5(\mathrm{a}, \mathrm{b})$ \\
\cline { 2 - 4 } $11-12$ weeks & $30(\mathrm{a})$ & $6(\mathrm{a}, \mathrm{b})$ & $12(\mathrm{~b})$ \\
\cline { 2 - 3 } 13 w or after & $4(\mathrm{a})$ & $0(\mathrm{a})$ & $0(\mathrm{a})$ \\
*Different letter shows statistical significance between groups in post-hoc analysis
\end{tabular}

Also, the decision of optimal time of surgery evaluated among surgeons was not different between surgical oncologists and general surgeons favoring 7-8 weeks and 11-12 weeks at the same degree. $(p=0,98)$ Forty-two percent of the surgeons declared they will use neoadjuvant chemotherapy between CRT or short-term radiotherapy and surgery. The utility of chemotherapy cycles was equal in ' 3 ' and ' 4 or more' as $17,1 \%$ among surgeons. Fifty-one percent of the surgeons do not offer chemotherapy until the time of the surgery. There was a significant difference between groups in posthoc analysis. (Table-3)

Table-3: The offered chemotherapy cycles before surgery according to groups.

\begin{tabular}{|c|c|c|c|}
\hline Offered Cycles & Medical Oncologists & Radiation Oncologists & Surgeons \\
\hline 0 & $0(\mathrm{a})$ & $22(b)$ & $18(b)$ \\
\hline 1 & 47 (a) & $0(b)$ & $3(a, b)$ \\
\hline 2 & 20 (a) & $8(a)$ & $2(\mathrm{a})$ \\
\hline 3 & $63(a)$ & $2(b)$ & $6(a, b)$ \\
\hline 4 or more & $54(a)$ & $4(\mathrm{a})$ & $6(\mathrm{a})$ \\
\hline Total & 184 & 36 & 35 \\
\hline
\end{tabular}

There was no difference among groups between surgery and the 'watch \& wait' strategy. $(p=0,11)$ A significant difference was observed between groups in offered neoadjuvant chemotherapy regimens. $(p<0,001)($ Table-4) 
Table-4: The offered chemotherapy regimens according to groups.

\begin{tabular}{|c|c|c|c|}
\hline Offered Regimen & Medical Oncologists & Radiation Oncologists & Surgeons \\
\hline Capecitabine/ 5-FU & 17 (a) & $5(a, b)$ & $13(b)$ \\
\hline$X E L O X$ & $93(a)$ & $8(b)$ & $1(\mathrm{c})$ \\
\hline Folfox & 45 (a) & $3(\mathrm{a})$ & $8(a)$ \\
\hline None & 29 (a) & $20(b)$ & $13(b)$ \\
\hline
\end{tabular}

\section{Discussion}

In our study, we evaluate similarities and differences in neoadjuvant treatment of rectal cancer according to radiotherapists, medical oncologists, and surgeons. The ultimate goal for neo-adjuvant treatment is CR which can be achieved in different treatment models for different risk stratification.

In patients who had pCR after neoadjuvant CRT, long-term outcome was reported to be excellent with less local and distant recurrence. The PCR rates were considered to be between $15-27 \%$ after neoadjuvant CRT and delayed surgery.[21] Although pCR is considered to be good prognostic factor 5 years OS is still the main determiner in this patient group.[22] Valentini et al showed that 2 years' DFS considered to be a better prognostic factor than $\mathrm{pCR}$ in rectal cancer.[23] The clinic utility of the $\mathrm{pCR}$ is still controversial and needs to be investigated. In contrast to other studies, our study population was formed by high numbers of CR patients, which established a valuable source of information in this patient group. Also, the study may be valuable for future meta-analysis.

The response to the CRT may have a relation with the delay of surgery.[24] The first strong study was the Lyon study showing six weeks of delay until the surgery increased the PCR in patients compared to two weeks.[25] In a large study, 10-11 weeks delay of surgery after neoadjuvant CRT have the highest PCR rates. No increased response rates were observed with waiting longer than this time interval.[26] Retrospective data reported prolonging the interval between CRT and surgery increasing the CR rates. Moore et al and Tulchinsky et al. declared waiting more than 7 weeks increased the CR rates significantly. $[24,27]$ Another study confirmed the prior studies with an eight-week waiting period. The CR rates were doubled with a longer interval.[28] This data was strengthened with a meta-analysis that was performed in 2005. Better outcomes and CR rates were reported without significant morbidity.[29] On the other hand, waiting longer than 11 weeks did not result in a favorable outcome. Comparing 7 weeks to 11 weeks interval between CRT and surgery was failed to show increased CR rates.[30] Similar to our results in a Turkish population study there was no difference between 4 and 8 weeks' waiting period between CRT and surgery.[31] In our study, we showed most of the participants had preferred 7-8 $8^{\text {th }}$ and $11-12^{\text {th }}$ weeks were the optimal operation period. Although the hypothesis of a longer waiting period increased the CR 
rates the optimal duration of the interval is not firmly established. A very small subset of the study population declared, they prefer the perform surgery more than the $13^{\text {th }}$ week. Also, the effect of genetic and racial differences on tumor response is not known. The confirmative results of Saglam et al. may strengthen the racial effect on tumor response in the Turkish population.[31] Our findings were substantially identical to the findings of the Canadian surgeon research in terms of optimal surgery time. [16] A higher rate of surgery before 6 weeks was seen in this trial, which might be related to short-course radiation treatment without chemotherapy. Also, there was no difference between surgeons, oncologists, and radiotherapists. A different trial which was based on a scenario-based questionnaire found a difference between decisions of the optimal time of surgery among surgeons, gastrointestinal oncologists, and radiotherapists. This study was focused only $6^{\text {th }}$ and $8^{\text {th }}$ weeks after CRT.[32]

The second controversial area is adding neoadjuvant chemotherapy to the treatment plan. Also, the optimal protocol and number of cycles are questionable. Garcia-Aguelar showed that adding two cycles of chemotherapy including 5-FU, oxaliplatin and leucovorin had increased the pCR rates up to $38 \%$. In our study, more than $20 \%$ of the patients received neoadjuvant chemotherapy. There was no effect on the prognosis in terms of OS. Also, the chemotherapy protocols and cycles were not eligible.[33] The neoadjuvant chemotherapy preference was significantly high in medical oncologists when compared with surgeons and radiotherapists. In the study Lefevre et al., although there were differences according to groups, long-term CRT was preferred in the absence of contraindications. The addition of neoadjuvant chemotherapy was not a frequent option for three groups consisting of surgeons, gastrointestinal oncologists, and radiotherapists.[32] Compatible with our results Hazen et. al reported long-term CRT with or without radiation boost is the most preferred option in colorectal surgeons according to their study. This study was consisting of colorectal surgeons different from our study. [17]

The selection of the treatment strategy is mainly dependent on primary risk factors and post-surgical margins. In the very-low risk group which is evaluated with endoscopic ultrasonography, the main treatment option is considered to be primary surgery. In patients with low-risk short-course, RT and conventional long-course radiotherapy with concurrent chemotherapy have similar results.[34] However, recent data published if the post-operative margin is at risk conventional treatment had similar results with short-course radiotherapy followed by pre-operative oxaliplatin including chemotherapy.[35] The difference between medical oncologists and other groups may depend on altered recurrence concerns in groups. In a study investigating treatment preferences of radiation oncologists, most of the physicians still prefer long-course radiation therapy. Short-course radiation therapy was mainly preferred in patients who are not a candidate for chemotherapy or social barriers for long-course treatment.[36]

The 'Watch \& Wait' strategy was less frequently selected in our study group. (17\%) The study which was published by Crawford et al., found 4.6 percent of participants select 'Watch \& Wait', nearly 54.6 percent declared case by case strategy selection while 40.9 percent favored surgery.[16] In a study investigating radiation oncologists, the 'Watch \& Wait' strategy was consisting 46 percent in correlation with the OnCoRe trial.[37] 


\section{Limitations}

The study was a survey study that tries to evaluate the pitfalls but was still lacking revealing most of the unidentified parts in daily practice. The study was designed to evaluate routine practice and hybrid methods in the treatment of rectal cancer which may lack enough quality to determine special situations such as total neo-adjuvant treatment, CRM positivity, or utility of neoadjuvant chemotherapy regimens such as FOLFOXIRI. The participants from radiotherapists and surgeons were lesser compared with medical oncologists which made comparison hard between groups. Also, high numbers of young participants in the medical oncology group may affect the results, this phenomenon was a result of an increased quota of medical oncology compared to surgical and radiation oncology. There were limited data in literature comparing medical oncologists, radiation oncologists, and surgeons which made comparison difficult with other studies.

\section{Conclusion}

In our study, we found the new pre-operative chemotherapy regimen with short-course radiotherapy was slowly adopted in current practice. Also, medical oncologists tend pre-operative chemotherapy compared with other groups. The optimal surgery time for patients receiving neoadjuvant treatment is still controversial.

\section{Learning Points}

Medical oncologists are more prone to up-front chemotherapy in rectal cancer.

Total neo-adjuvant treatment is not a highly-selected option.

Medical oncologists offer more chemotherapy cycles before surgery.

'Watch \& Wait' strategy is still not an option for all groups.

\section{Declarations}

\section{Acknowledgments}

Ismail Beypınar: Idea, Concept, Manuscript Writing, Statistical Analysis Mustafa Tercan \& Fuzuli Tugrul: Data Collection, Revision, Literature Search

The authors declare no conflict of interest.

This research did not receive any specific grant from funding agencies in the public, commercial, or notfor-profit sectors. 


\section{References}

1. Sauer R, Liersch T, Merkel S, et al (2012) Preoperative versus postoperative chemoradiotherapy for locally advanced rectal cancer: Results of the German CAO/ARO/AIO-94 randomized phase III trial after a median follow-up of 11 years. Journal of Clinical Oncology 30:1926-1933. https://doi.org/10.1200/JCO.2011.40.1836

2. Peeters KCMJ, Marijnen CAM, Nagtegaal ID, et al (2007) The TME trial after a median follow-up of 6 years: Increased local control but no survival benefit in irradiated patients with resectable rectal carcinoma. Annals of Surgery 246:693-701. https://doi.org/10.1097/01.sla.0000257358.56863.ce

3. Beypinar I, Demir H, Araz M, et al (2020) The View of Turkish Oncologists Regarding MSI Status and Tumor Localization in Stage II and III Colon Cancer. Journal of Gastrointestinal Cancer. https://doi.org/10.1007/s12029-020-00542-5

4. Zhou ZR, Liu SX, Zhang TS, et al (2014) Short-course preoperative radiotherapy with immediate surgery versus long-course chemoradiation with delayed surgery in the treatment of rectal cancer: A systematic review and meta-analysis. Surgical Oncology 23:211-221

5. Martin ST, Heneghan HM, Winter DC (2012) Systematic review and meta-Analysis of outcomes following pathological complete response to neoadjuvant chemoradiotherapy for rectal cancer. British Journal of Surgery 99:918-928

6. An X, Lin X, Wang FH, et al (2013) Short term results of neoadjuvant chemoradiotherapy with fluoropyrimidine alone or in combination with oxaliplatin in locally advanced rectal cancer: A meta analysis. European Journal of Cancer 49:843-851. https://doi.org/10.1016/j.ejca.2012.09.026

7. Benson AB, Venook AP, Cederquist L, et al (2017) Colon cancer, version 1.2017: Clinical practice guidelines in oncology. JNCCN Journal of the National Comprehensive Cancer Network 15:370-398

8. van Gijn W, Marijnen CAM, Nagtegaal ID, et al (2011) Preoperative radiotherapy combined with total mesorectal excision for resectable rectal cancer: 12-year follow-up of the multicentre, randomised controlled TME trial. The Lancet Oncology 12:575-582. https://doi.org/10.1016/S1470-2045(11)70097-3

9. Yeo SG, Kim DY, Kim TH, et al (2010) Pathologic complete response of primary tumor following preoperative chemoradiotherapy for locally advanced rectal cancer: Long-term outcomes and prognostic significance of pathologic nodal status (KROG 09-01). Annals of Surgery.

https://doi.org/10.1097/SLA.0b013e3181f3f1b1

10. Francois Y, Nemoz CJ, Baulieux J, et al (1999) Influence of the interval between preoperative radiation therapy and surgery on downstaging and on the rate of sphincter-sparing surgery for rectal cancer: The Lyon R90-01 randomized trial. Journal of Clinical Oncology.

https://doi.org/10.1200/JC0.1999.17.8.2396

Page 10/13 
11. SUIT HD, GALLAGER HS (1964) Intact Tumor Cells in Irradiated Tissue. Archives of pathology 78:648-651

12. Evans J, Tait D, Swift I, et al (2011) Timing of surgery following preoperative therapy in rectal cancer: The need for a prospective randomized trial? Diseases of the Colon and Rectum 54:1251-1259. https://doi.org/10.1097/DCR.0b013e3182281f4b

13. Saglam S, Bugra D, Saglam EK, et al (2014) Fourth versus eighth week surgery after neoadjuvant radiochemotherapy in T3-4/N0+ rectal cancer: Istanbul R-01 study. Journal of Gastrointestinal Oncology. https://doi.org/10.3978/j.issn.2078-6891.2013.025

14. Lefevre JH, Mineur L, Kotti S, et al (2016) Effect of interval (7 or 11 weeks) between neoadjuvant radiochemotherapy and surgery on complete pathologic response in rectal cancer: A multicenter, randomized, controlled trial (GRECCAR-6). Journal of Clinical Oncology 34:3773-3780. https://doi.org/10.1200/JC0.2016.67.6049

15. Terzi C, Bingul M, Arslan NC, et al (2020) Randomized controlled trial of 8 weeks' vs 12 weeks' interval between neoadjuvant chemoradiotherapy and surgery for locally advanced rectal cancer. Colorectal Disease. https://doi.org/10.1111/codi.14867

16. Crawford A, Firtell J, Caycedo-Marulanda A (2019) How Is Rectal Cancer Managed: a Survey Exploring Current Practice Patterns in Canada. Journal of Gastrointestinal Cancer 50:260-268. https://doi.org/10.1007/s12029-018-0064-9

17. Hazen SM, Sluckin T, Beets G, et al (2021) Current practices concerning the assessment and treatment of lateral lymph nodes in low rectal cancer: a survey among colorectal surgeons in The Netherlands. Acta Chirurgica Belgica. https://doi.org/10.1080/00015458.2021.2016204

18. Zhang C, Ye F, Liu Y, et al (2018) Morphologic predictors of pathological complete response to neoadjuvant chemoradiotherapy in locally advanced rectal cancer. Oncotarget 9:4862-4874. https://doi.org/10.18632/oncotarget.23419

19. Beypinar I, Araz M, Uysal M, Yalcin S (2019) First-line treatment choices of Turkish medical oncologists in metastatic colorectal cancer: A survey study. Journal of B.U.ON. 24:68-76

20. Beypinar I, Demir H, Araz M, et al (2020) The View of Turkish Oncologists Regarding MSI Status and Tumor Localization in Stage II and III Colon Cancer. Journal of Gastrointestinal Cancer. https://doi.org/10.1007/s12029-020-00542-5

21. Maas M, Nelemans PJ, Valentini V, et al (2010) Long-term outcome in patients with a pathological complete response after chemoradiation for rectal cancer: A pooled analysis of individual patient data. The Lancet Oncology 11:835-844. https://doi.org/10.1016/S1470-2045(10)70172-8 
22. Petrelli F, Borgonovo K, Cabiddu M, et al (2017) Pathologic complete response and disease-free survival are not surrogate endpoints for 5-year survival in rectal cancer: An analysis of 22 randomized trials. Journal of Gastrointestinal Oncology 8:39-48. https://doi.org/10.21037/jgo.2016.11.03

23. Valentini V, van Stiphout RGPM, Lammering G, et al (2015) Selection of appropriate end-points ( $p C R$ vs $2 y D F S$ ) for tailoring treatments with prediction models in locally advanced rectal cancer. Radiotherapy and Oncology 114:302-309. https://doi.org/10.1016/j.radonc.2015.02.001

24. Moore HG, Gittleman AE, Minsky BD, et al (2004) Rate of Pathologic Complete Response with Increased Interval between Preoperative Combined Modality Therapy and Rectal Cancer Resection. Diseases of the Colon and Rectum 47:279-286

25. Francois Y, Nemoz CJ, Baulieux J, et al (1999) Influence of the interval between preoperative radiation therapy and surgery on downstaging and on the rate of sphincter-sparing surgery for rectal cancer: The Lyon R90-01 randomized trial. Journal of Clinical Oncology 17:2396-2402. https://doi.org/10.1200/jco.1999.17.8.2396

26. Sloothaak DA, Geijsen DE, van Leersum NJ, et al (2013) Optimal time interval between neoadjuvant chemoradiotherapy and surgery for rectal cancer. The British journal of surgery 100:933939. https://doi.org/10.1002/bjs.9112

27. Tulchinsky H, Shmueli E, Figer A, et al (2008) An interval $>7$ weeks between neoadjuvant therapy and surgery improves pathologic complete response and disease-free survival in patients with locally advanced rectal cancer. Annals of Surgical Oncology 15:2661-2667. https://doi.org/10.1245/s10434008-9892-3

28. Kalady MF, de Campos-Lobato LF, Stocchi L, et al (2009) Predictive factors of pathologic complete response after neoadjuvant chemoradiation for rectal cancer. Annals of Surgery 250:582-588. https://doi.org/10.1097/SLA.0b013e3181b91e63

29. Petrelli F, Coinu A, Lonati V, Barni S (2015) A systematic review and meta-analysis of adjuvant chemotherapy after neoadjuvant treatment and surgery for rectal cancer. International Journal of Colorectal Disease 30:447-457

30. Lefevre JH, Mineur L, Kotti S, et al (2016) Effect of interval (7 or 11 weeks) between neoadjuvant radiochemotherapy and surgery on complete pathologic response in rectal cancer: A multicenter, randomized, controlled trial (GRECCAR-6). Journal of Clinical Oncology 34:3773-3780. https://doi.org/10.1200/JC0.2016.67.6049

31. Saglam S, Bugra D, Saglam EK, et al (2014) Fourth versus eighth week surgery after neoadjuvant radiochemotherapy in T3-4/N0+ rectal cancer: Istanbul R-01 study. Journal of Gastrointestinal Oncology 5:9-17. https://doi.org/10.3978/j.issn.2078-6891.2013.025 
32. Lefevre $\mathrm{JH}$, Benoist S (2017) Practice patterns for complex situations in the management of rectal cancer: A multidisciplinary inter-group national survey. Journal of Visceral Surgery 154:147-157. https://doi.org/10.1016/j.jviscsurg.2016.08.012

33. Garcia-Aguilar J, Chow OS, Smith DD, et al (2015) Effect of adding mFOLFOX6 after neoadjuvant chemoradiation in locally advanced rectal cancer: A multicentre, phase 2 trial. The Lancet Oncology 16:957-966. https://doi.org/10.1016/S1470-2045(15)00004-2

34. Erlandsson J, Holm T, Pettersson D, et al (2017) Optimal fractionation of preoperative radiotherapy and timing to surgery for rectal cancer (Stockholm III): a multicentre, randomised, nonblinded, phase 3, non-inferiority trial. The Lancet Oncology 18:336-346. https://doi.org/10.1016/S14702045(17)30086-4

35. Bujko K, Wyrwicz L, Rutkowski A, et al (2016) Long-course oxaliplatin-based preoperative chemoradiation versus $5 \times 5$ Gy and consolidation chemotherapy for cT4 or fixed cT3 rectal cancer: Results of a randomized phase III study. Annals of Oncology 27:834-842. https://doi.org/10.1093/annonc/mdw062

36. Mowery YM, Salama JK, Zafar SY, et al (2017) Neoadjuvant long-course chemoradiation remains strongly favored over short-course radiotherapy by radiation oncologists in the United States. Cancer 123:1434-1441. https://doi.org/10.1002/cncr.30461

37. Yahya J, Herzig D, Farrell M, et al (2018) Survey results of US radiation oncology providers' contextual engagement of watch-and-wait beliefs after a complete clinical response to chemoradiation in patients with local rectal cancer. Journal of Gastrointestinal Oncology 9:1127-1132. https://doi.org/10.21037/jgo.2018.08.02

\section{Supplementary Files}

This is a list of supplementary files associated with this preprint. Click to download.

- survey.docx 